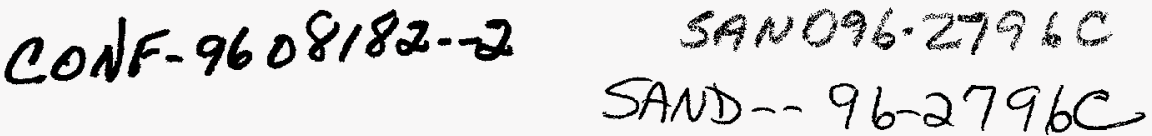

\section{Design, Modelling, and Current Interpretations of the H-19 and H-11 Tracer Tests at the WIPP Site}

\author{
Lucy C. Meigs, Richard L. Beauheim and James T. McCord \\ Sandia National Laboratories, USA \\ Yvonne W. Tsang \\ E. O. Lawrence Berkeley National Laboratory, USA \\ Roy Haggerty \\ Oregon State University, USA

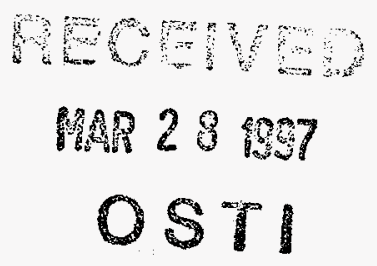

Abstract

Site-characterization studies at the Waste Isolation Pilot Plant (WIPP) site in southeastern New Mexico, USA identified ground-water flow in the Culebra Dolomite Member of the Rustler Formation as the most likely geologic pathway for radionuclide transport to the accessible environment in the event of a breach of the WIPP repository through inadvertent human intrusion. The Culebra is a 7-m-thick, variably fractured dolomite with massive and vuggy layers. In the 1980s, a series of tracer tests was performed in the Culebra to identify important transport mechanisms and quantify transport parameters for use in a preliminary performance assessment (PA) of the WIPP site. Comments received from numerous review and regulatory groups indicated the need to distinguish among alternative conceptual models. Based on extensive interactions with numerous review groups and outside scientists, additional testing was planned.

The results of recent tracer tests, as well as hydraulic tests, laboratory measurements, and reexamination of Cuelbra geology and stratigraphy, have led to a significant refinement of the conceptual model for transport in the Culebra. The Culebra was previously conceptualized as a medium where advection was only through fractures with diffusion into a relatively homogeneous rock matrix. The Culebra is now conceptualized as a heterogeneous medium with multiple scales of porosity. Tracer test results and geologic observations suggest that flow occurs within fractures, and to some extent within interparticle porosity and vugs connected by microfractures. Diffusion occurs within all connected porosity. Numerical simulations suggest that the data from the tracer tests cannot be simulated with heterogeneous single-porosity models; significant matrix diffusion appears to be required. The low permeability and lack of significant tracer recovery from tracers injected into the upper Culebra suggest that transport primarily occurs in the lower Culebra.

The success of the recent tracer tests in refining the conceptual model of Culebra transport was due to extensive interactions between modellers and experimenters prior to and during the tests. The test design was the direct result of interpretations of past tests and was continuously refined during the tests as additional insight was gained from evaluation and interpretation of new data. This step-wise approach was valuable for designing a robust test, but the approach could be improved by adopting an even more evolutionary strategy over a longer time frame.

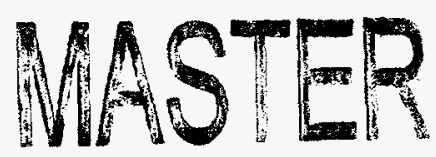




\section{DISCLAIMER}

This report was prepared as an account of work sponsored by an agency of the United States Government. Neither the United States Government nor any agency thereof, nor any of their employees, make any warranty, express or implied, or assumes any legal liability or responsibility for the accuracy, completeness, or usefulness of any information, apparatus, product, or process disclosed, or represents that its use would not infringe privately owned rights. Reference herein to any specific commercial product, process, or service by trade name, trademark, manufacturer, or otherwise does not necessarily constitute or imply its endorsement, recommendation, or favoring by the United States Government or any agency thereof. The views and opinions of authors expressed herein do not necessarily state or reflect those of the United States Government or any agency thereof. 


\section{DISCLAMMIER}

Portions of this document may be illegible in electronic image products. Images are produced from the best available original document. 


\section{Introduction}

The Waste Isolation Pilot Plant (WIPP) is a proposed repository for transuranic wastes constructed in bedded Permian-age halite deposits in southeastern New Mexico, USA. Sitecharacterization studies at the WIPP site identified ground-water flow in the Culebra Dolomite Member of the Rustler Formation as the most likely geologic pathway for radionuclide transport to the accessible environment in the event of a breach of the WIPP repository through inadvertent human intrusion. The Culebra is a 7 -m-thick, variably fractured dolomite with massive and vuggy layers. Between 1980 and 1988, tracer tests were performed in the Culebra at five locations (H-2, H-3, H-4, H-6, and H-11 hydropads) to identify important transport processes and mechanisms, and to quantify transport parameters. The information derived from these tests was used in preliminary performance assessments (PA) of the WIPP and was presented to numerous review and regulatory groups, including the International Project to Study Validation of Geosphere Transport (INTRAVAL), the U.S. National Academy of Sciences, the U.S. Environmental Protection Agency (EPA), and the New Mexico Environmental Evaluation Group (EEG). Comments received from these groups indicated the need for additional tracer tests to distinguish among possible alternative conceptual models, define transport processes and parameters more definitively, and develop a defensibly conservative simplified model of transport for PA. Through extensive interactions with these review groups and other interested scientists, a plan for tracer testing at a new test site, the H-19 hydropad, was developed. Based on the results of preliminary tests at $\mathrm{H}-19$, additional testing was planned and performed at the $\mathrm{H}-11$ hydropad to resolve questions associated with tests previously conducted at that location. Beauheim et al. [1] describe the rationale for additional experiments in greater detail.

The objectives of the new experiments were to test important features of the transport models used to interpret the previous tests, further evaluate transport processes in the Culebra, and provide quantitative estimates of important transport parameters. This new information was to be combined with the information derived from previous tracer tests and laboratory tests (e.g., porosity measurements, batch tests, etc.) to develop both a transport model and parameter ranges for use in PA. The initial Culebra transport model was based on interpretations of previous tracer tests performed at the $\mathrm{H}-3, \mathrm{H}-6$, and $\mathrm{H}-11$ hydropads. These interpretations were based on a double-porosity model in which advective transport occurred through uniformly spaced fractures (constant matrix block size), with physical retardation provided by diffusion from the fractures into cubic matrix blocks, and directional differences in transport were caused by anisotropy in permeability. The new tests were designed to evaluate the assumptions used in this transport model. Specifically, the tests should demonstrate whether matrix diffusion occurs in the Culebra, whether an idealized fracture-matrix geometry was adequate to model test results, whether anisotropy alone could account for directional differences in transport, and the effect that layering has on transport within the Culebra.

Three elements in the design of the new tests were focused on the demonstration of matrix diffusion. First, single-well injection-withdrawal (SWIW) tracer tests were planned that were expected to distinguish clearly between the effects of matrix diffusion and heterogeneity in permeability. Second, tracer injections were to be repeated in convergent-flow tests performed at different pumping rates to show the effects of advective residence time on diffusion. Third, two different conservative tracers having different free-water diffusion coefficients were to be injected together in a convergent-flow tracer test to show the effects of different amounts of diffusion. See Beauheim et al. [1] for a discussion of pre-test calculations for these three design elements.

\section{Results of Recent Tests}

Testing began at the H-19 hydropad in June 1995 with SWTW and preliminary convergentflow tests after wells $\mathrm{H}-19 \mathrm{~b} 0, \mathrm{~b} 2, \mathrm{~b} 3$, and $\mathrm{b} 4$ had been completed (Figure 1). The purpose of the 
preliminary convergent-flow test was to provide site-specific data for the determination of locations of three additional wells, and to provide data for test design refinements and model testing. The breakthrough curves obtained from the convergent-flow test showed transport to be slower at $\mathrm{H}-19$ than at other sites tested. As a result, additional wells were drilled closer to $\mathrm{H}-19 \mathrm{b0}$ than had been projected without site-specific data. Numerical simulation indicated that the preliminary convergent-flow test data could not be matched with the range of parameters and conceptual model used to match tracer tests conducted previously at other sites. To match the preliminary test data approximately, the advective porosity had to be greater than 0.03 , which is larger than typical fracture porosities. This result raised questions concerning the validity of conceptualizing the Culebra as a medium where advection was only through fractures with diffusion into a relatively homogeneous rock matrix. The results of the preliminary test

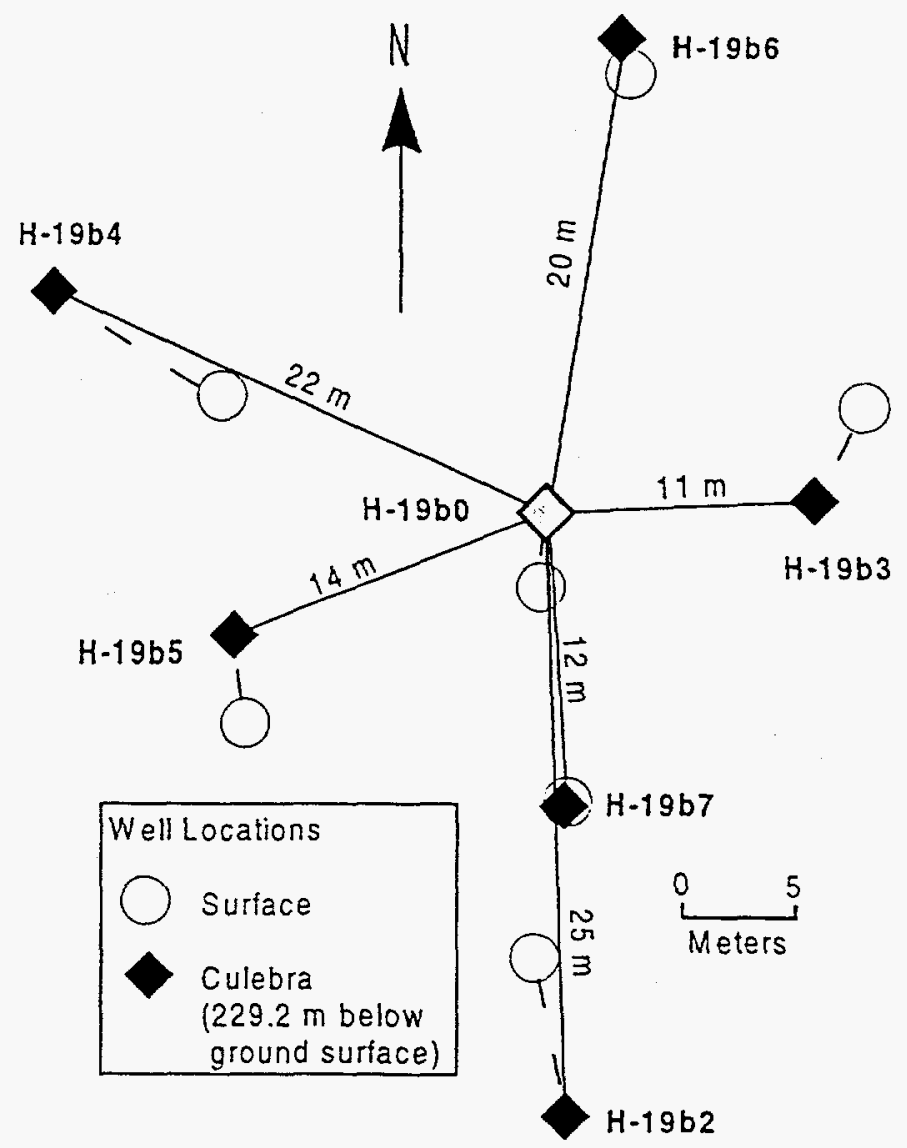

TRI.6115.48:-2

Figure 1. Well locations at the H-19 hydropad. also led to refinements in the design of tests performed after all seven wells had been completed, as well as to the decision to perform additional tests at the previously tested $\mathrm{H}-11$ hydropad (Figure 2) where significantly faster transport had been observed. Conducting a simpler and shorter preliminary tracer test prior to the subsequent complicated and lengthy tracer test provided valuable information for refinement of test design, refinement of conceptual models, and provided a 'dry run' for testing equipment and procedures used in the field and laboratory.

Starting in October 1995, hydraulic tests were performed in the seven wells at the H-19 hydropad. These tests revealed that the permeability of the upper portion of the Culebra was significantly lower than that of the lower Culebra at this site [1]. The information obtained from the hydraulic tests was used to refine the tracer-test design. Tracer testing resumed at H-19 in December 1995, with a SWIW test of the lower Culebra followed by convergent-flow testing with tracer injections in six wells. At three wells, tracers were injected into both the entire thickness of the Culebra and into upper and lower portions of the Culebra. Some of the flow paths were tested again beginning in February 1996 after the pumping rate had been reduced. Testing at H-11 began in February 1996 and consisted of a SWTW test over the entire thickness of Culebra, followed by convergent-flow tests involving two injection wells and two pumping rates.

The breakthrough curves for the six pathways tested with the multi-well convergent-flow test at $\mathrm{H}-19$ show significant variations (Figure 3). For example, the fastest peak arrival time is not 


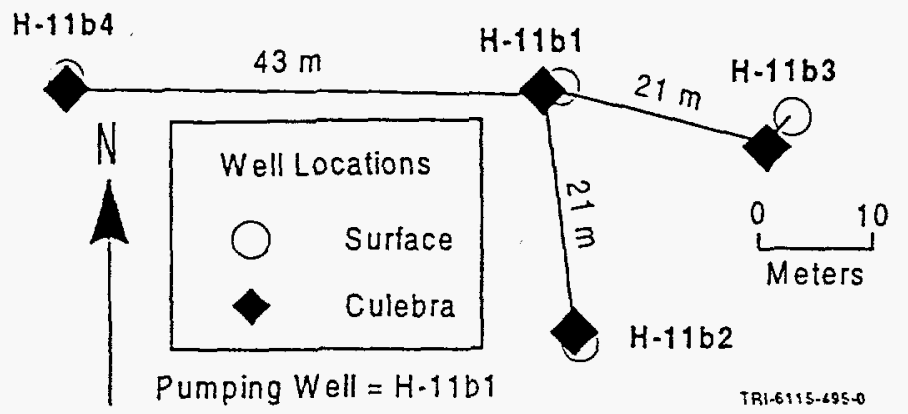

Figure 2. Well locations at the $\mathrm{H}-11$ hydropad.

from the shortest travel distance and the slowest peak arrival time is not from the longest travel distance, indicating that transport is through a heterogeneous and/or anisotropic medium. The peak heights of breakthrough curves at $\mathrm{H}-11$ and H-19 for given pathways (e.g., $\mathrm{H}-11 \mathrm{~b} 3$ to $\mathrm{H}-11 \mathrm{~b} 1)$ for the two different pumping rates used are the same within measurement error (Figure 4). Tracers injected into the upper portion of the Culebra at $\mathrm{H}-19$ were only detected at the pumping well in very low concentrations. The iodide data from the three multi-well injections where iodide and a benzoic acid were injected simultaneously were difficult to obtain and less reliable due to the high salinity of the Culebra brine. Despite these difficulties, the normalized concentration breakthrough curves, at all three locations, suggest that the peak height of the iodide was less than the peak height of the benzoic acid, as would be expected if matrix diffusion is occurring because iodide has a larger free-water diffusion coefficient than the benzoic acid. The difference between the iodide data and the benzoic acid data is greater at $\mathrm{H}-11$ (Figure 4) than at $\mathrm{H}-19$ (Figure 3).

The data from the breakthrough curves and subsequent modelling results (e.g. the large advective porosities that appear to be necessary to model the tracer breakthrough data from $\mathrm{H}-19$ ) motivated refinement of the conceptualization of transport in the Culebra. Through careful reexamination of the geology and stratigraphy of the Culebra, a more thorough conceptualization has

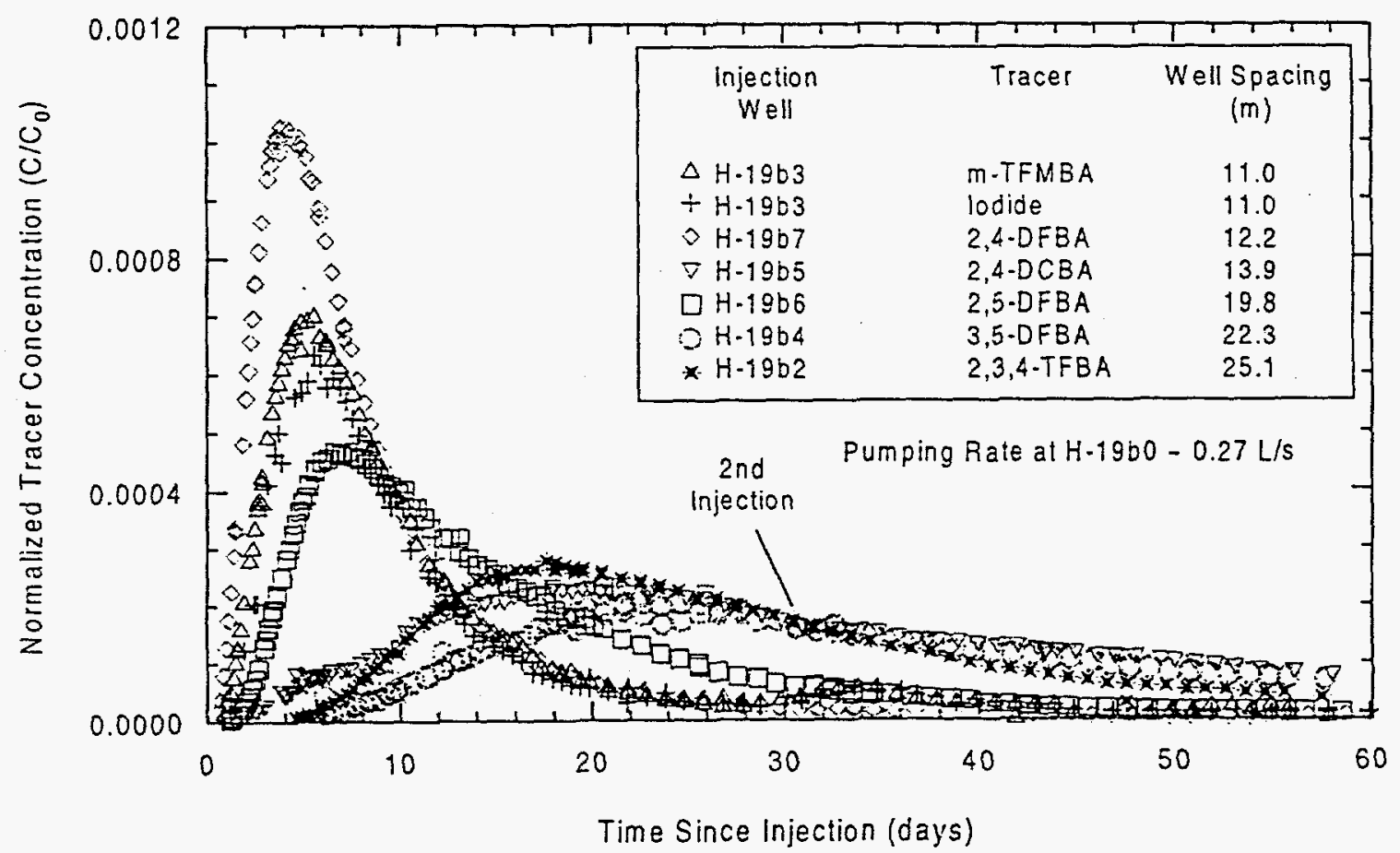

TR1.6115-496-0

Figure 3. Observed tracer data (e.g., 2,3,4-trifluorobenzoic acid) for the H-19 multi-well convergent-flow test at the high pumping rate. 


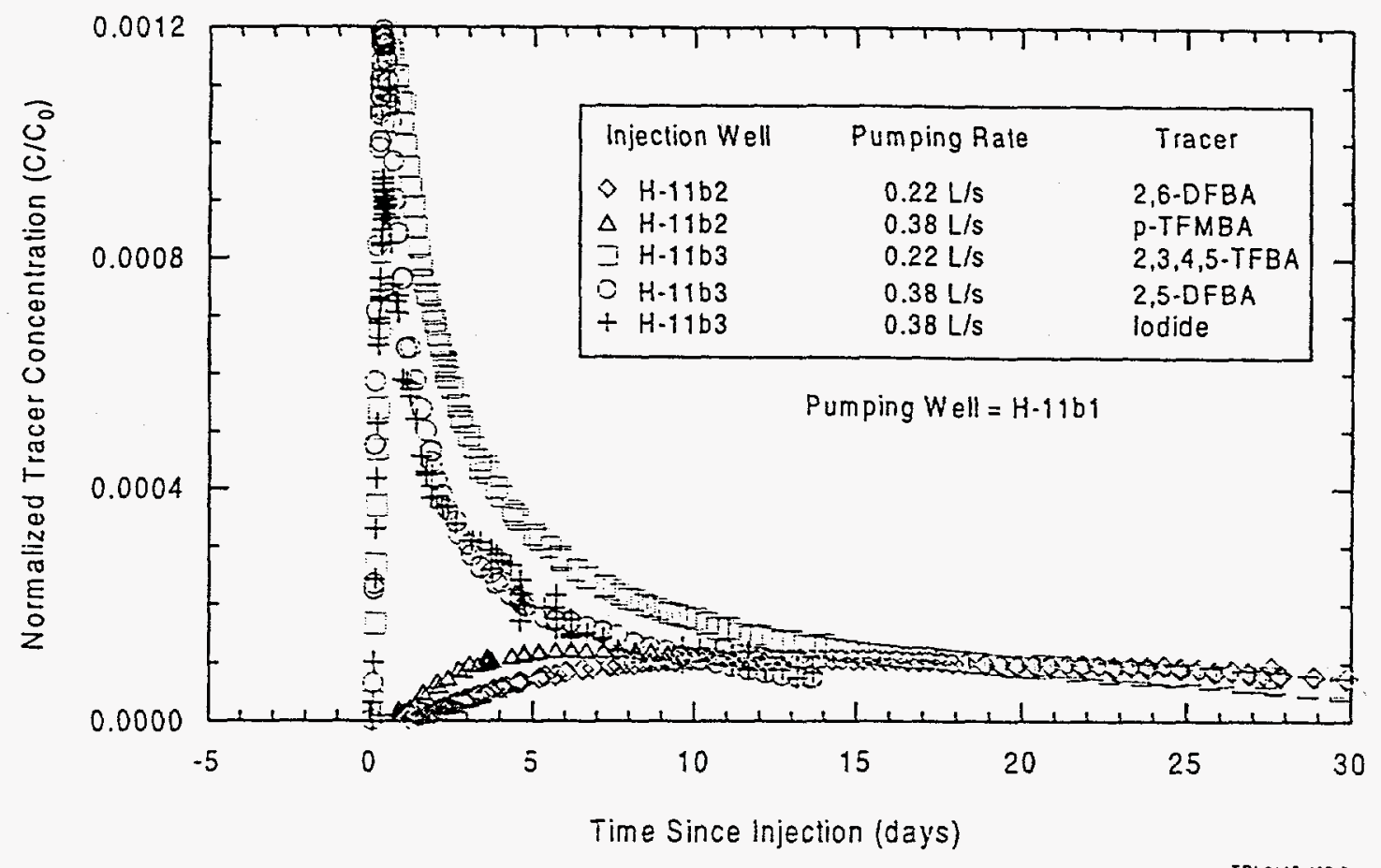

Figure 4. Observed tracer data for the $\mathrm{H}-11$ multi-w'ell convergent-flow test at the high and low pumping rates.

been developed of the important processes that control transport. The Culebra has non-uniform properties both horizontally and vertically as was demonstrated by both hydraulic and tracer tests. The low permeability and lack of significant tracer recovery from injections into the upper portion of the Culebra suggest that transport primarily occurs in the lower Culebra. Multiple scales of porosity exist within the Culebra, including fractures ranging from microscale to large, vuggy zones, intercrystalline, interparticle and intraparticle porosity (Figure 5). Laboratory (core plug) measurements of porosity of the Culebra yield values between 0.03 to 0.30 (median of 0.16 ), which indicates that there is significant porosity for advection and diffusion. Tracer test results and geological observations suggest flow can occur within fractures, and to some extent within interparticle porosity and vugs where they are connected by fractures. Diffusion occurs within all connected porosity and will be the dominant transport mechanism in relatively low permeability portions of the formation. The variation in peak arrival times in tracer-breakthrough curves between the $\mathrm{H}-11$ and the $\mathrm{H}-19$ hydropads suggests that the types of porosity contributing to rapid advective transport may vary spatially.

\section{Numerical Simulations of Tracer-Test Data}

Interpretations of the tracer-test data completed to date have relied on both homogeneous and heterogeneous single- and double-porosity continuum models. In the double-porosity models, the Culebra is conceptualized as two continua: the advective porosity (fractures, vugs, and possibly interparticle porosity) where flow is the dominant process and the diffusive porosity (all other connected porosity) where diffusion is the dominant process. Spatial variations in advective transport are represented in numerical simulations of tracer-test data with unconditioned, spatially correlated, random hydraulic conductivity fields. Diffusive transport was simulated with double-porosity models using both a single rate of diffusion (which conceptualizes diffusion as a homogeneous process) and multiple rates of diffusion (which conceptualizes the diffusive porosity as heterogeneous). The multi- 


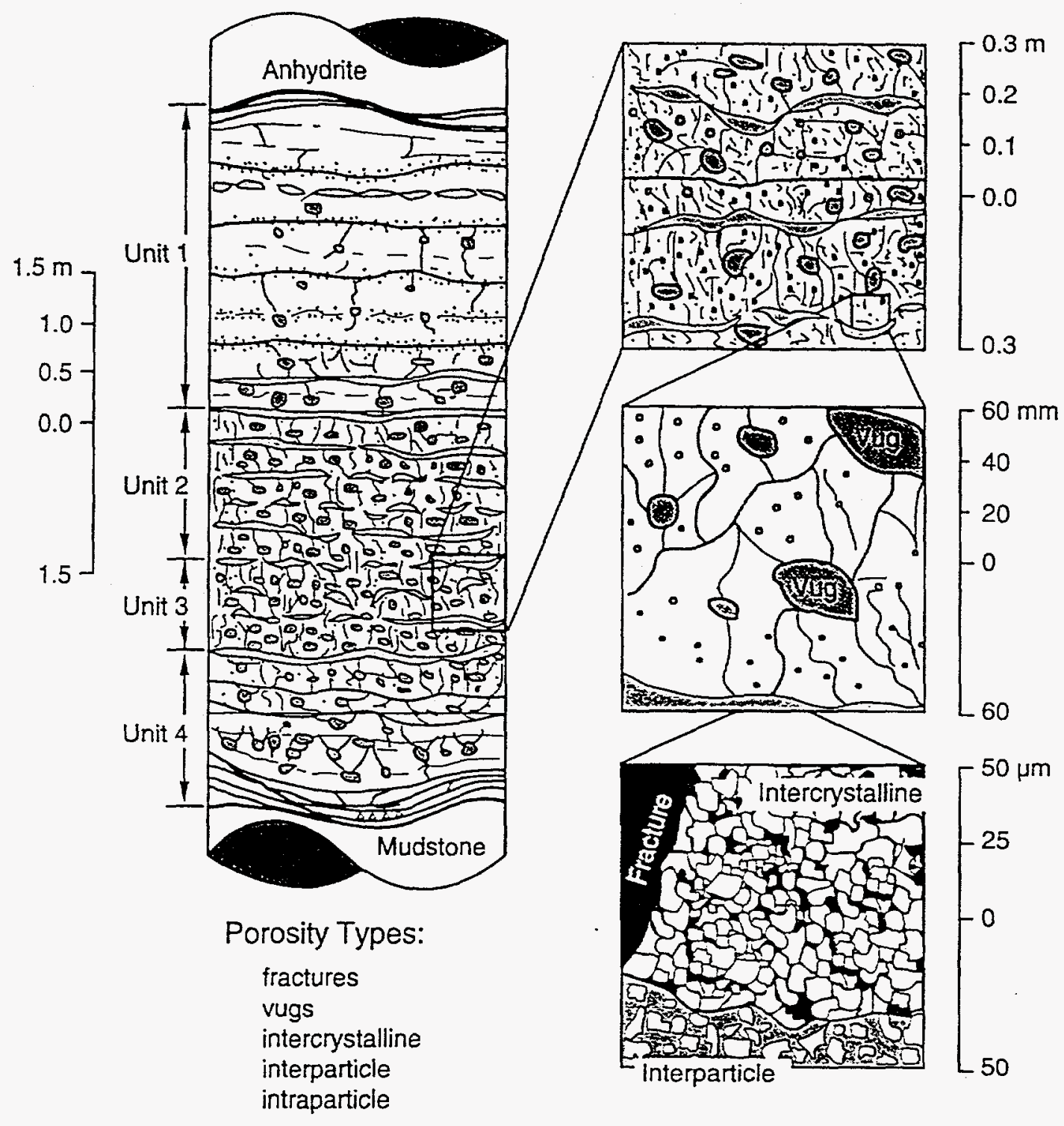

TRI-6115-5140

Figure 5. Schematic illustration showing multiple scales of Culebra porosity.

rate model employed [2] represents the heterogeneities in block size (surface area for diffusion and diffusion distance) and the tortuous nature of the pore structure with a distribution of diffusion rates (Figure 6).

Interpretations completed to date have shown that the SWIW test data from both the H-11 and $\mathrm{H}-19$ hydropads cannot be explained by heterogeneity in hydraulic conductivity alone. Simulations of cumulative mass recovery during the withdrawal phase of the tracer test with homogeneous and heterogeneous single-porosity models suggest that mass recovery for these conceptual models should be very rapid. Figure 7 shows the mass recovery from the H-11 SWTW test compared to heterogeneous single- and double-porosity simulations. The H-11 (and H-19) data show a slow mass recovery as would be anticipated if some form of diffusional process were controlling mass 
recovery. Simulations further suggest that the SWIW data cannot be adequately explained using a single-rate double-porosity model with homogeneous or heterogeneous permeability fields. With a single-rate double-porosity model, the simulated tracer recovery concentration during a SWTW test will asymptotically approach a constant slope of $-3 / 2$ on a $\log -\log$ plot of normalized tracer concentration vs. elapsed time (Figure 8). If matrix blocks are small and the solute diffuses to the centre of the block, the diffusion rate will decrease and the late-time slope will steepen. The late-time slope of the data from the $\mathrm{H}-11$ and two H-19 SWTW tests was approximately $-5 / 2$ rather than $-3 / 2$. An excellent fit to the data can be obtained using the multi-rate diffusion model that incorporates a statistical distribution of mass transfer rates. Figure 9 show's fits to both the $\mathrm{H}-11$ and H-19 SWIW test data with the multi-rate model. Figure 10 show's the distributions of diffusion rates that were used to match the data in Figure 9. For these distributions, if tortuosity is assumed to be constant at 0.1 , the mean block sizes would be $0.03 \mathrm{~m}$ and $0.006 \mathrm{~m}$ for the $\mathrm{H}-11$ and $\mathrm{H}-19$ simulations, respectively. These diffusion rate distributions cannot be considered to represent unique solutions because equally good fits could be obtained using different values of advective and diffusive porosity that would produce somewhat different distributions.

The multi-well convergent-flow data also appear to exhibit evidence of heterogeneity and matrix diffusion. Preliminary attempts to match the convergentflow data with a single anisotropy were unsuccessful. Heterogeneity, as opposed to simple anisotropy, appears to be required to capture the variations in the six tracertest breakthrough curves. Numerical simulations of tracer-test data with heterogeneous single and doubleporosity models suggested that the data cannot be adequately modelled without matrix diffusion (Figure 11). Heterogeneous single-porosity models cannot simultaneously match the magnitude of the peak concentration and peak arrival time. Heterogeneous single-rate double-porosity models can match individual breakthrough curves quite well. However, when the parameters used to match data from the high pumping rate $(0.27 \mathrm{~L} / \mathrm{s})$ are used to simulate the breakthrough curve for the low pumping rate $(0.16 \mathrm{~L} / \mathrm{s})$, the match is not satisfactory (Figure 12). With a single-rate double-porosity model with a given pumping rate, as matrix block size decreases, peak heights gradually first decrease and then increase (Figure 13). The increase in peak height occurs as a result of solute diffusion reaching the centre of the matrix block, which causes a decrease in the concentration gradient and, therefore, a decrease in the diffusion rate. As a result, peak heights will not always be lower for lower pumping rates. Simulations have shown that a model with multiple rates of diffusion can produce peak heights that are similar for the two pumping rates used for the H-19 testing (Figure 14). 


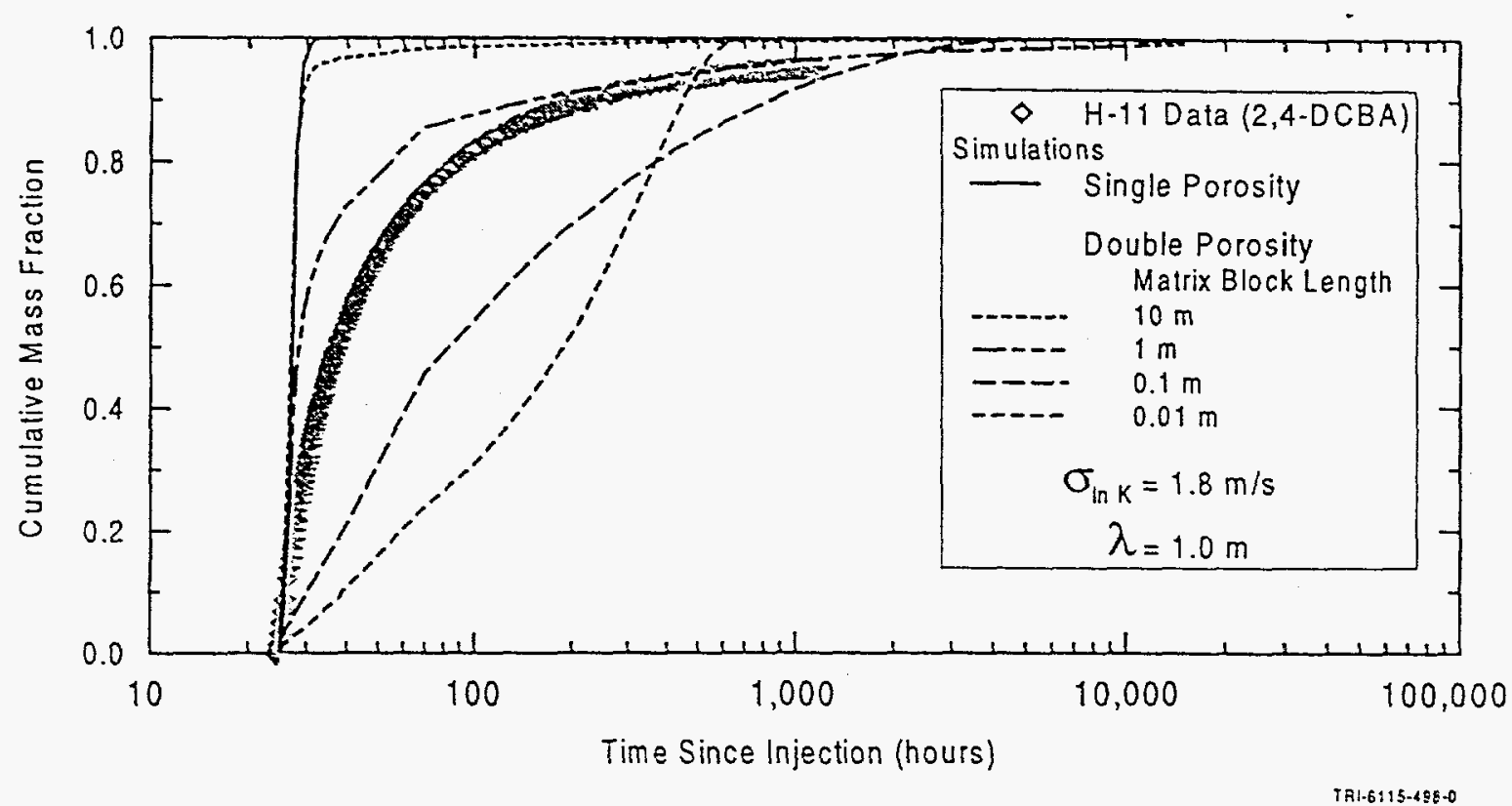

Figure 7. Simulated mass recovery curves for H-11 SWIW test with single-porosity and doubleporosity models compared to observed data. For the simulations, the heterogeneous field of hydraulic conductivity had a standard deviation $(\sigma)$ in natural $\log$ space of $1.8 \mathrm{~m} / \mathrm{s}$ and an exponential model with a correlation length $(\lambda)$ of $1.0 \mathrm{~m}$.

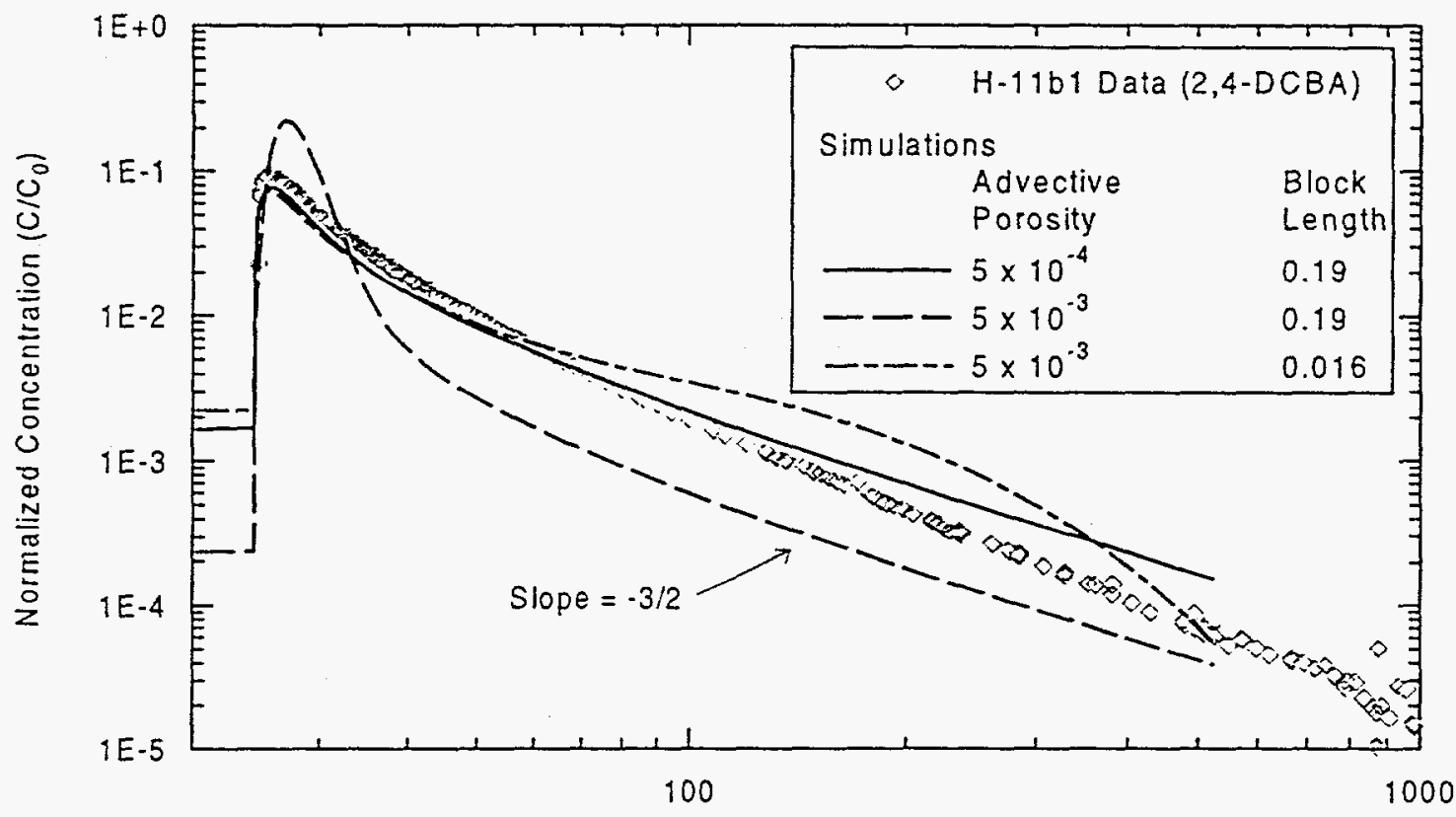

Time Since Injection of First Tracer Slug (hours)

TR1.6115-498.0

Figure 8. Simulated concentration vs. time curves for H-11 SWIW test with the single-rate double-porosity model compared to observed data. 


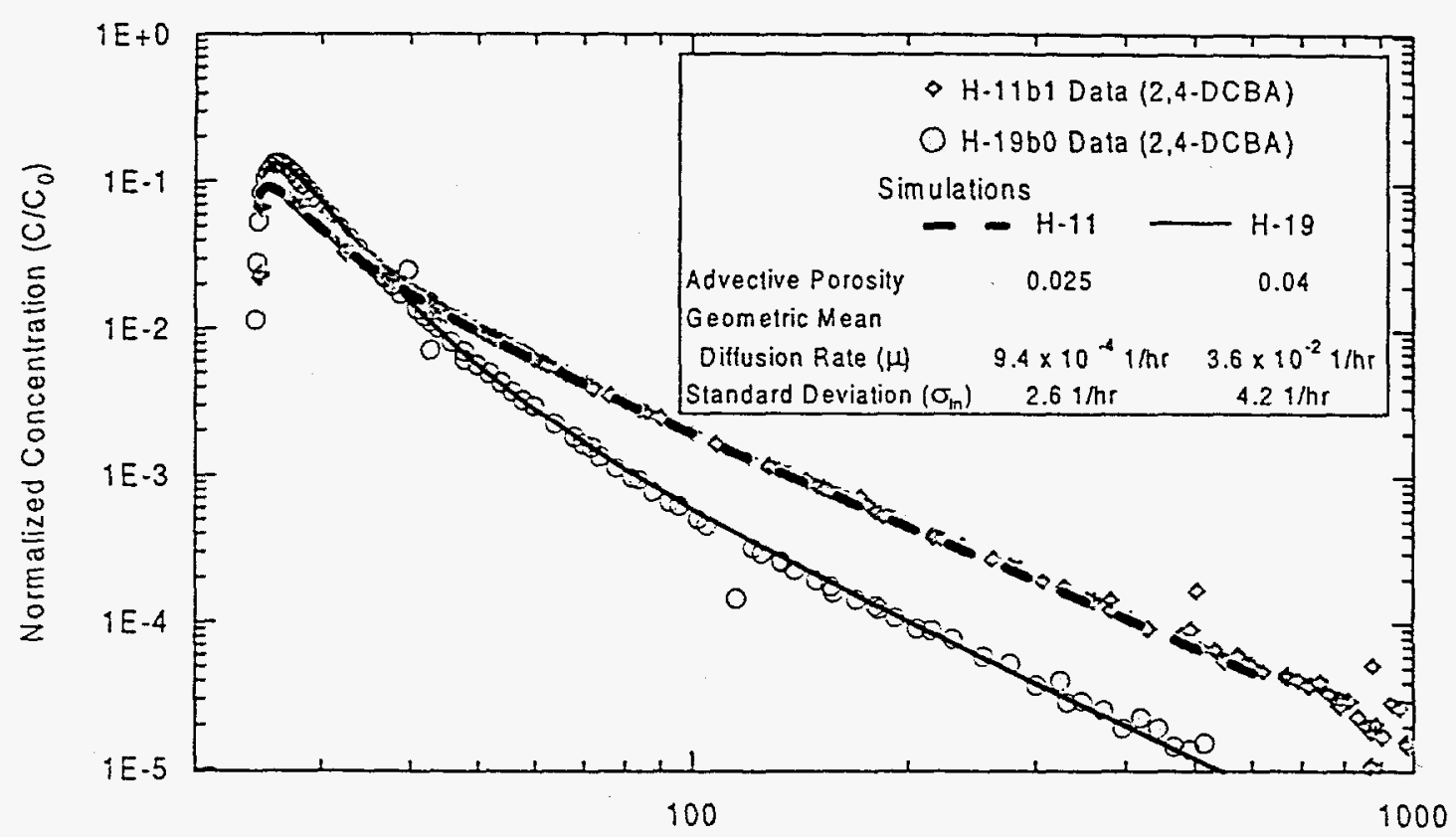

Time Since Injection of First Tracer Slug (hours)

TRI.6115.500.0

Figure 9. Simulated concentration vs. time curves for H-11 and H-19 SWIW tests with the multi-rate double-porosity model compared to observed data.

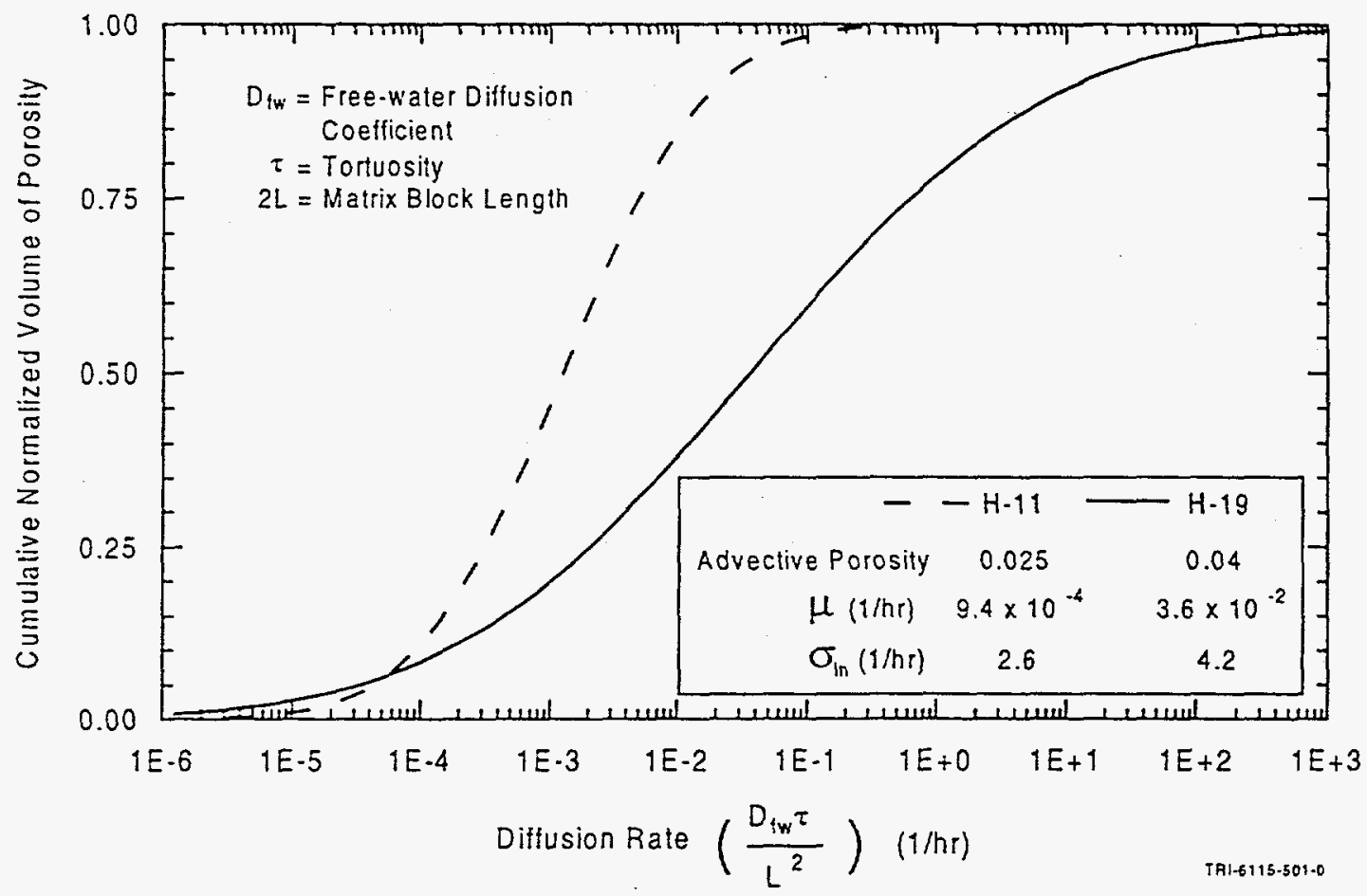

Figure 10. Diffusion rate coefficient cumulative distribution function for $\mathrm{H}-11$ and $\mathrm{H}-19 \mathrm{SWIW}$ test simulations shown in Figure 9. 


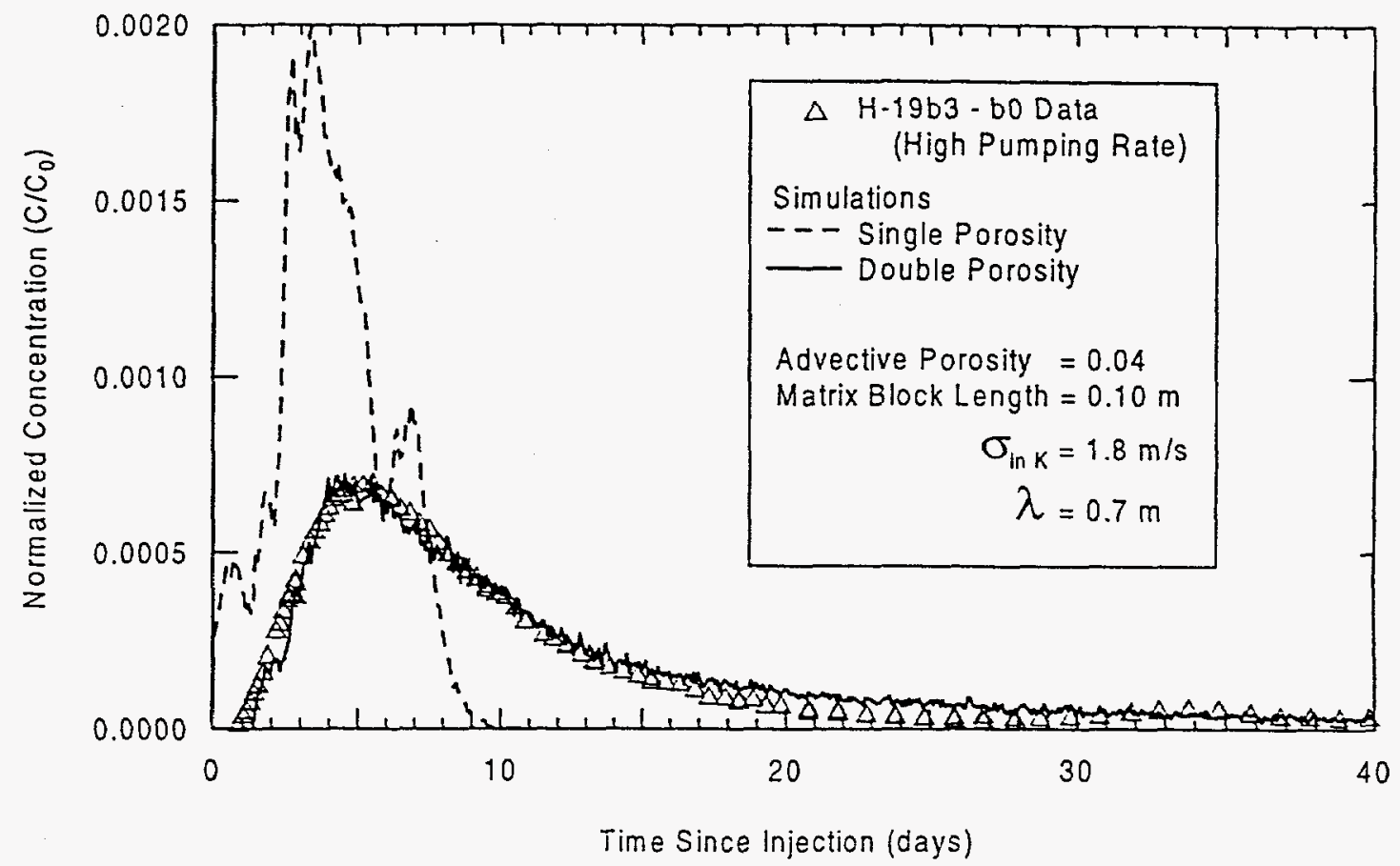

Figure 11. Simulated breakthrough curves for multi-well convergent-flow test data, $\mathrm{H}-19 \mathrm{~b} 3$ - b0 path, using heterogeneous, single- and double-porosity models.

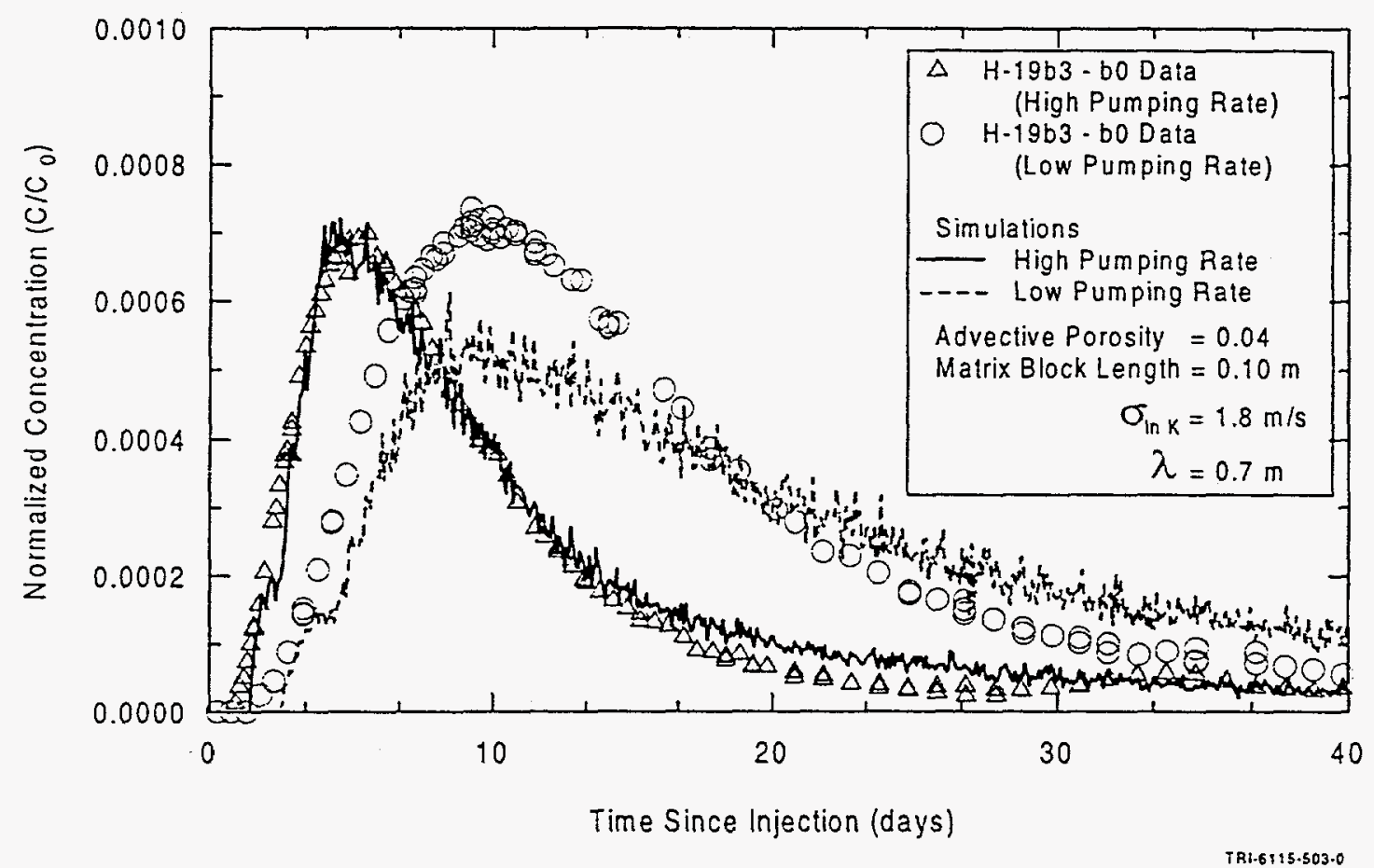

Figure 12. Simulated breakthrough curves for multi-well convergent-flow test data, $\mathrm{H}-19 \mathrm{~b} 3$ - b0 path, using heterogeneous, single-rate double-porosity model for two different pumping rates. 


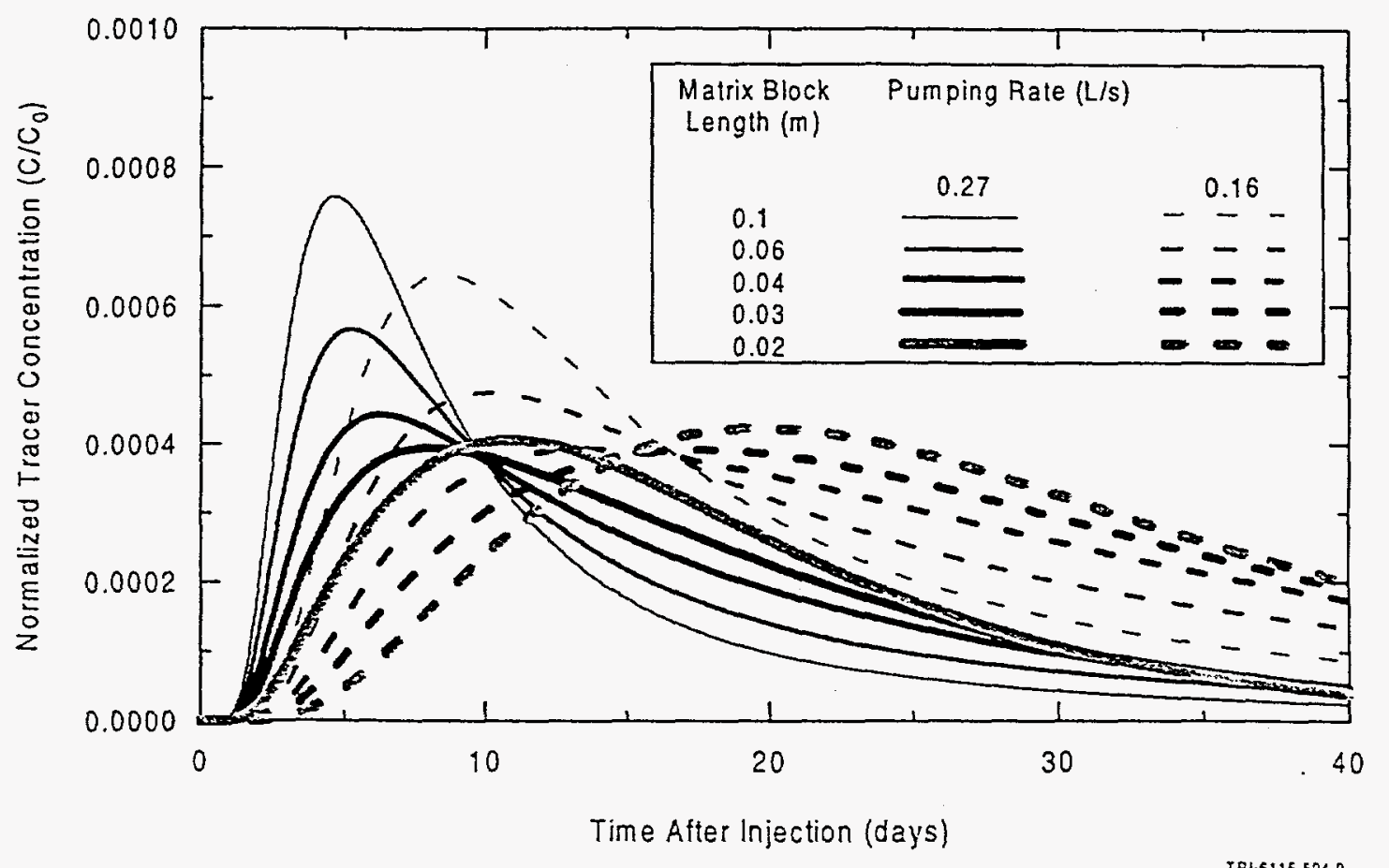

Figure 13. Effect of pumping rate and block size on peak height and breakthrough time.

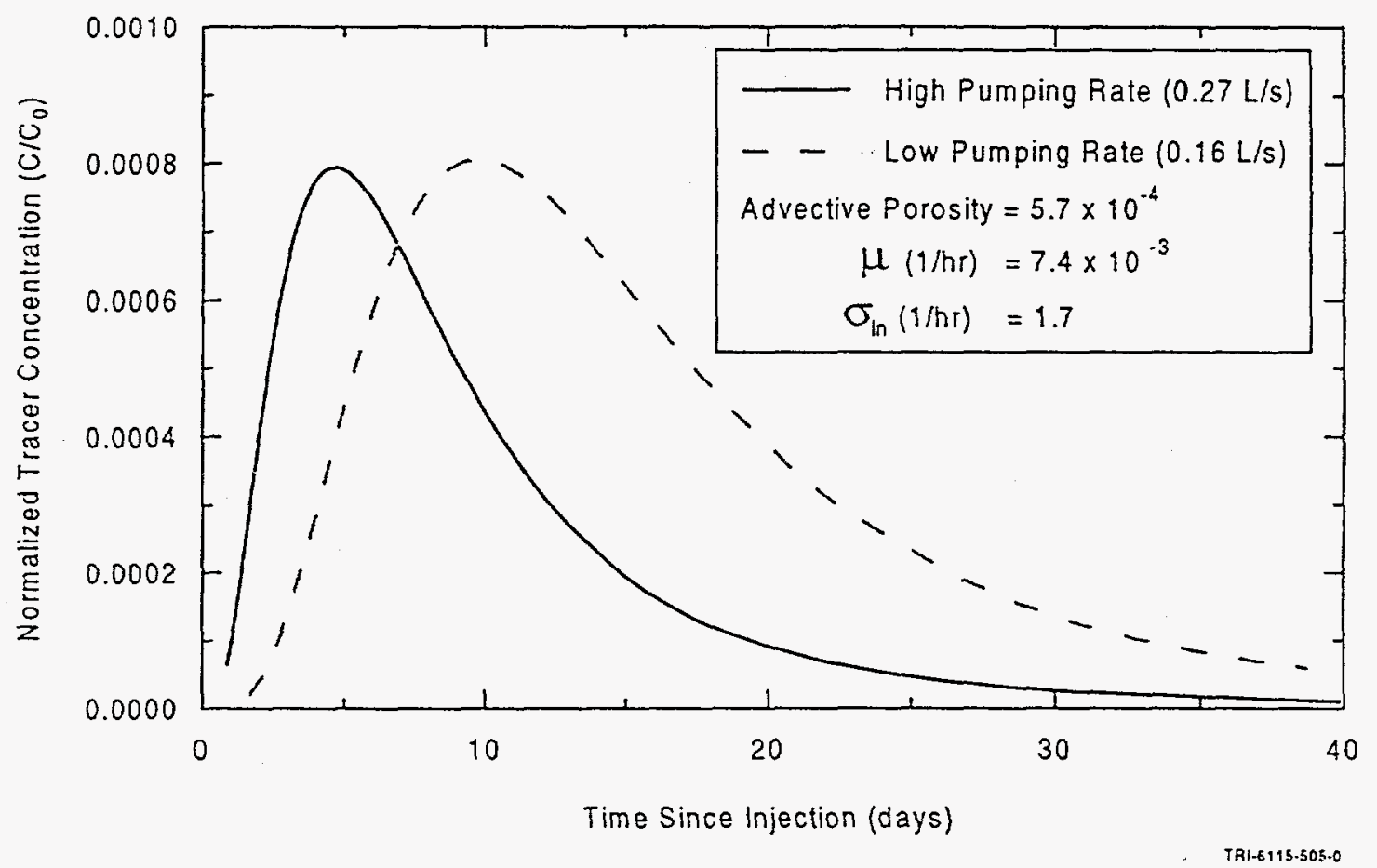

Figure 14. Simulations of two different pumping rates with multi-rate double-porosity model. 
The data collected from recent tracer tests and numerical simulations to date have led to a significant refinement of the conceptual model of the Culebra. Numerical simulations of the multiwell convergent-flow and SWIW tests suggest that the data cannot be simulated with heterogeneous single-porosity models; significant matrix diffusion appears to be required. Numerical simulations of the multi-well tracer tests suggest that advective transport is not limited to fractures and that there is significant variation in advective porosity across the WIPP site.

\section{Discussion}

For the larger-scale simulations conducted for PA, spatial variability in advective transport is represented by heterogeneous transmissivity fields that have been conditioned on available point transmissivity data and transient pressure data from large scale pumping tests. In the PA calculations, the lower permeability of the upper portion of the Culebra has been approximated by eliminating this portion of the Culebra from the transport model. Numerical simulations of tracer test data were used to bound transport parameters for use in PA models. Possible spatial variability in transport properties (diffusion and sorption rates) has not been treated explicitly in the PA model to date. Attempts have been made to take this variability into account by providing PA with conservative ranges of values for transport parameters (i.e., values that could lead to greater releases than expected).

Tracer tests appear to be well suited for providing insight into the important processes that are operative at a given site and for testing conceptual models. For example, the mass-recovery curve from the SWIW test appears to be well suited to evaluating the importance of diffusive processes. Complex tests are valuable for evaluating conceptual models. A clear conceptual model that incorporates the tracer test results with what is understood about the geology and stratigraphy at the PA scale is important if tracer test results are to be used to provide transport properties over a much larger region. Tracer tests will always have limitations: they cannot directly test the materials for the length and time scales of interest for PA calculations. Thus, one must evaluate whether altemative conceptual models can explain the data, particularly if different conceptual models would lead to different results at the PA scale.

The success of the recent tracer tests in refining the conceptual model of Culebra transport were the result of extensive interaction between the modellers and the experimenters prior to and during the tests. The details of the design were continuously refined during the tests as additional insight was gained from evaluation and interpretation of new data. The iterative evaluation of test data was especially important for such test-design features as injection into different layers of the Culebra and the selection of different pumping rates.

The testing approach used could be improved by adopting a more evolutionary strategy over a longer time frame. The step-wise strategy that was used, which included conducting a preliminary tracer test prior to completion of all wells, was very valuable for developing a robust tracer-test design. Ideally, field testing would begin after only one or a very few wells were installed. The early tests would be completely interpreted before plans for additional wells and tests were finalized. In parallel with field tests, detailed laboratory tests would be performed. The laboratory tests would provide a controlled environment for testing rock diffusion and sorption properties. The later field tests and well locations would be designed to address questions/ambiguities raised by the early tests in both the field and lab. New models/hypotheses raised by the new tests would be evaluated through tests at other locations. Design of new tests would be well integrated with ongoing laboratory programs. Sorbing tracers would be added to the programme at a late stage to evaluate conclusions reached from laboratory testing. Specific experimental enhancements would include increasing the contrasts in pumping rates and possibly tracer diffusion coefficients, improved control over rates/pressures of tracer injection to allow interpretation of hydraulic responses, and development of a 
reliable means of determining the downhole tracer source term. Laboratory testing and modelling would be used to provide confidence that the downhole source term could actually be measured under the anticipated test conditions before investing in a field-scale tool and field testing.

\section{Summary}

Extensive interactions between experimenters and modellers resulted in a robust test design, which lead to significant refinement in the conceptual model for transport in the Culebra. Based on hydraulic tests, tracer tests, and geologic descriptions, the Culebra is now conceptualized as a heterogeneous medium with multiple scales of porosity. The low permeability and lack of significant tracer recovery from tracers injected into the upper Culebra suggest that transport primarily occurs in the lower Culebra. The tracer test data provide evidence that transport is not limited to fractures. Numerical simulations of the multi-well convergent-flow tracer test suggest that advective transport is not limited to fractures. Advection also appears to occur to some extent through interparticle porosity and vugs connected by microfractures. Numerical simulations of the multi-well and SWIW tests suggest that the data cannot be simulated with heterogeneous single-porosity models; significant matrix diffusion appears to be required. Numerical simulations of the tracer test data with this refined conceptual model were used to bound transport parameters for use in PA models.

\section{Acknowledgments}

This work was supported by the US Department of Energy (US DOE) under contract DEAC04-94AL85000. Sean McKenna of Sandia National Laboratories did the simulations in Figures 11 and 12. Joanna Ogintz and Toya Jones of INTERA did the simulations in Figures 8 and 13. Robert Holt provided the geologic information for creation of Figure 5. Numerous members of the Sandia National Laboratories and INTERA staff helped to make these tracer tests possible. Sandia is a multiprogram laboratory operated by Sandia Corporation, a Lockheed Martin Company, for the US Department of Energy. References

[1] Beauheim, R.L., Meigs, L.C., and Davies, P.B., Rationale for the H-19 and H-11 Tracer Tests at the WIPP Site, 1996, [this volume].

[2] Haggerty, R., and Gorelick, S.M., Multiple-rate mass transfer for modeling diffusion and surface reactions in a media with pore-scale heterogeneity, Water Resources Research, vol. 31, no. 10, p. 2383-2400, 1995.

[3] Hadermann, J., Heer W., The Grimsel (Switzerland) migration experiment: integrating field experiments, laboratory investigations and modelling, Joumal of Contaminant Hydrology, vol. 21, p. 87-100, 1996. 